\title{
A longitudinal study characterising a large adult primary ciliary dyskinesia population
}

\author{
Anand Shah ${ }^{1,2}$, Amelia Shoemark ${ }^{2,3}$, Stephanie J. MacNeill ${ }^{4}$, Basrull Bhaludin ${ }^{5}$, \\ Andrew Rogers ${ }^{1,3}$, Diana Bilton ${ }^{1,2}$, David M. Hansell ${ }^{2,5}$, Robert Wilson ${ }^{1,2}$ and \\ Michael R. Loebinger ${ }^{1,2}$
}

Affiliations: ${ }^{1}$ Host Defence Unit, Royal Brompton and Harefield NHS Foundation Trust, London, UK. ${ }^{2}$ Imperial College London, London, UK. ${ }^{3}$ Electron Microscopy Dept, Royal Brompton and Harefield NHS Foundation Trust, London, UK. "'Dept of Occupational and Environmental Medicine, Imperial College London, London, UK. ${ }^{5}$ Dept of Radiology, Royal Brompton and Harefield NHS Foundation Trust, London, UK.

Correspondence: Michael R. Loebinger, Host Defence Unit, Royal Brompton and Harefield NHS Foundation Trust, Sydney Street, London, SW3 6NP, UK. E-mail: M.LoebingerQrbht.nhs.uk

ABSTRACT Primary ciliary dyskinesia (PCD) in adults has not been well described. In this retrospective observational study we aimed to characterise a large adult population and identify features associated with disease progression.

We retrospectively analysed 151 adult patients at a single tertiary centre at baseline and longitudinally for a median of 7 years.

We found significant variation in age at diagnosis (median 23.5 years; range $<1-72$ years). Older age at diagnosis was associated with impaired baseline forced expiratory volume in $1 \mathrm{~s}$ (FEV1) $(\mathrm{r}=-0.30, \mathrm{p}=0.01)$ and increased Pseudomonas aeruginosa colonisation (difference in medians 17 years (95\% CI 4.5-20 years); $\mathrm{p}=0.002$ ). Lung function decline was estimated at FEV1 decline of $0.49 \%$ predicted per year. Lung function decline was associated with ciliary ultrastructure, with microtubular defect patients having the greatest decline $(\mathrm{p}=0.04)$. High-resolution computed tomography (HRCT) scores of severity of bronchial wall dilatation $(p<0.001)$ and extent of bronchiectasis $(p=0.03)$ additionally showed evidence of modifying FEV1 decline with age.

Our study reveals that a large proportion of adult PCD patients are diagnosed late, with impaired FEV1 and increased $P$. aeruginosa colonisation. Increased disease burden on HRCT and ciliary ultrastructure may predict progressive lung function decline. This study characterises a large adult PCD population, identifies features associated with disease progression and highlights the need for prospective trials to determine whether early diagnosis of high-risk subgroups alongside optimal management can modify disease progression.

@ERSpublications

Increased severity on HRCT and ciliary ultrastructure predict progressive lung function decline in adults with PCD http://ow.ly/4ncpnD

Editorial comment in: Eur Respir J 2016; 48: 297-300.

This article has supplementary material available from erj.ersjournals.com

Received: Sept 152014 | Accepted after revision: April 132016 | First published online: June 102016

Support statement: This study was supported by the National Institute for Health Research (NIHR) Respiratory Disease Biomedical Research Unit at the Royal Brompton and Harefield NHS Foundation Trust and Imperial College London. No external funding source was required for this study.

Conflict of interest: None declared.

Copyright OERS 2016 


\section{Introduction}

Primary ciliary dyskinesia (PCD) is a genetically heterogeneous disorder resulting in dysfunctional ciliary motility and structure. Clinical presentation is varied but characterised by neonatal respiratory distress and chronic oto-sino-pulmonary disease due to impaired mucociliary transport. Male infertility due to sperm flagella dysmotility is common and organ laterality occurs in $\sim 50 \%$ patients [1]. Adults with PCD have varied radiological severity of disease and lung function impairment $[2,3]$.

The progression of PCD in adults has not been well described. Previous studies have primarily focused on paediatric populations, which may not reflect progression in adult life and, importantly, do not include the significant proportion of patients with PCD that remain undiagnosed until adulthood $[2,4,5]$.

In a cross-sectional analysis of a paediatric population it was recently suggested that ciliary ultrastructure related to severity of disease, contradicting previous studies [4-6]. There are also conflicting data regarding the utility and transverse correlation of lung function and high-resolution computed tomography (HRCT) as measures of disease severity $[2,7,8]$. The data regarding longitudinal prognosis of PCD are limited, with small studies demonstrating correlation between HRCT structural lung disease and subsequent longitudinal lung function $[2,3]$.

In this retrospective observational study we aimed to describe and characterise a large adult PCD population, and explore features associated with disease progression and poor prognosis using longitudinal lung function. A better understanding of the pattern of disease progression and predictors of prognosis within this patient population may facilitate future focused research and identify patient subgroups that may benefit from intensive management to prevent lung function decline.

\section{Materials and methods}

All adult patients at a tertiary respiratory referral centre (Royal Brompton and Harefield NHS Foundation Trust, London, UK) with a multidisciplinary consensus diagnosis of PCD, including historical patients, were included in the study. Clinical history and nasal nitric oxide results were considered in conjunction with light microscopy and transmission electron microscopy (TEM) findings. A diagnosis of PCD was made when a compatible clinical history was accompanied by a recognised defect of ciliary beat and/or ultrastructure as previously described [9]. A breakdown of the diagnostic criteria is available in the supplementary material.

Clinical data were retrieved from medical records regarding age at diagnosis, sputum microbiology and longitudinal lung function. Lung function at time of diagnosis or transition to adult care was used to determine baseline. Smokers were those with $>5$-pack-year history. Chronic colonisation was defined as the isolation of potentially pathogenic bacteria or fungi in the sputum on two or more occasions at least 3 months apart in a 1-year period with $>50 \%$ positive cultures during the year [10]. All patients had three or more sputum cultures over the duration of follow-up. Nontuberculous mycobacteria (NTM) infection was defined according to the American Thoracic Society guidelines and allergic bronchopulmonary aspergillosis (ABPA) according to the British Thoracic Society guidelines [11, 12]. Sputum microbiology for patients is presented as cumulative colonisation over the duration of the follow-up period. Longitudinal lung function data were obtained from patients with at least two lung function records when clinically stable with a minimum of three forced expiratory manoeuvres within the same lung function laboratory in the absence of bronchodilator. Lung function decline was expressed as forced expiratory volume in $1 \mathrm{~s}$ (FEV1) \% predicted and mL-year ${ }^{-1}$ and estimated using Global Lung Function Initiative reference equations [13]. European Community for Steel and Coal reference equations were used for measurement of transfer factor of the lung for carbon monoxide (TLCO) [14]. Follow-up ranged from 1 to 34 years (median 7 years), during the period 1980-2014.

HRCT chest imaging suitable for scoring was assessed and scored by an independent chest radiologist using a method previously described $[15,16]$. Further details are provided in the supplementary material.

This study has been reviewed by the Royal Brompton Research Office and deemed not requiring of formal ethics approval under the Governance Arrangements for Research Ethics Committees.

\section{Statistical analysis}

Clinical and demographic data were expressed as frequencies, proportions, mean \pm SD or median with range, depending on the distribution. Continuous variables were compared between independent groups using the Mann-Whitney and Kruskal-Wallis tests. Spearman's rank correlation was used to assess for correlation between continuous measures. p-values of $<0.05$ were considered statistically significant.

To model the effects of HRCT score, ciliary ultrastructure and ciliary beat frequency on lung function decline, HRCT scores and ciliary ultrastructural defects were grouped into categories as described in the supplementary material. 
Apart from an assessment of baseline lung function, all analyses of FEV1 were performed using longitudinal measures. These were analysed using linear mixed models with random intercept allowing for repeated observations within patients. Age at the time of the FEV1 measure was included as a linear term and we fitted a random slope for this and used an unstructured covariance matrix. The other covariates considered for inclusion in the model were time-independent variables with the exception of body mass index (BMI) and Pseudomonas aeruginosa colonisation. We fitted separate models for each of the following covariates to see if they were associated with FEV1 independently of age using the likelihood ratio test: age at diagnosis, sex, $P$. aeruginosa colonisation, BMI, mean nitric oxide, ciliary beat frequency and TEM subtypes. Factors significantly associated with FEV1 $(\mathrm{p}<0.05)$ were retained in a core model. We then fitted models of FEV1 with each of the HRCT variables (in continuous form) as covariates (separately) adjusting for age and any factors identified above. We separately tested for interaction between HRCT factors and age, to assess whether the mean change in FEV1 with age was modified by HRCT score. To facilitate the description of any effect modification, where there was evidence of interaction $(\mathrm{p}<0.05$ by likelihood ratio test comparing a model with and without the interaction term), models were stratified on a categorised version of the HRCT variable (see supplementary table S1 for details).

Results from our models are presented as regression coefficients (with 95\% CI), except for age, where we presented the regression coefficient and used the between-individual slope variance to estimate the range between which 95\% of values would lie. Data were analysed using Graphpad (version 5.0; Graphpad Software Inc., La Jolla, CA, USA) and Stata (version 13; StataCorp LP, College Station, TX, USA).

\section{Results}

\section{Description of adult PCD population}

There were 151 adults with a multidisciplinary diagnosis of PCD. A full diagnostic breakdown of the patient population is presented in figure 1a. Patient demographics are shown in table 1.

The median age in the population was 35 years. Seven sibling pairs and one family with three siblings were included in the population. During the follow-up period, the incidence of all-cause mortality was $4.6 \%(n=7)$ with respiratory mortality of $3.3 \%(n=5)$. Non-respiratory death was due to metastatic carcinoma $(n=2)$. The median age of death was 65 years (range 31-75 years). No patients underwent lung transplantation.

HRCT was able to be scored in 93 patients, with some historic imaging unavailable. The timing of HRCT was at diagnosis in $40 \%(n=37)$, at transition to adult care in $15 \%(n=14)$ and during the follow-up period in $45 \%(n=42)$. The median age at HRCT was 33 years. Scoring revealed a wide range of extent of bronchiectasis, bronchial wall dilatation and thickness (table 1). Four (4.3\%) patients within the population had no evidence of bronchiectasis on HRCT imaging. One patient had a pneumonectomy and three had lobectomies. Figure $1 \mathrm{~b}$ shows the lobar distribution of bronchiectasis within the population, with a high prevalence of right middle lobe, lingular and basal bronchiectasis. There was no difference in lobar distribution of bronchiectasis according to PCD TEM ciliary ultrastructure (data not shown).
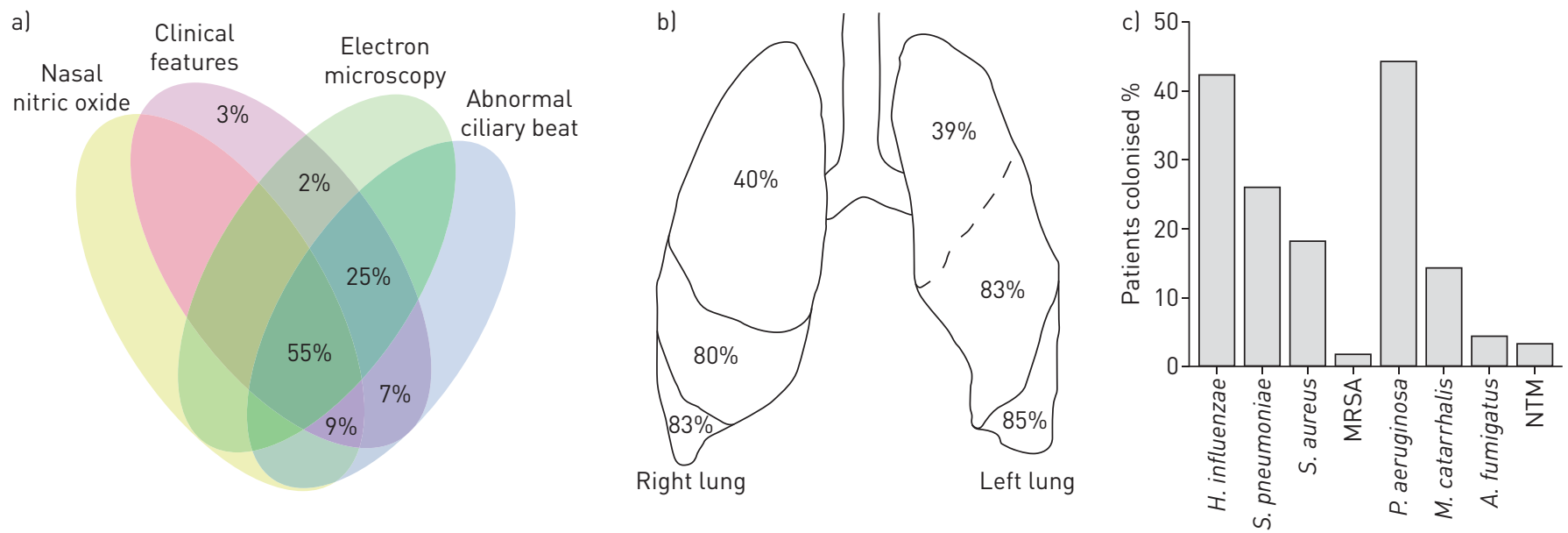

FIGURE 1 a) Diagnostic breakdown of our cohort. b) Lobar distribution of bronchiectasis seen in adult primary ciliary dyskinesia. c) Cumulative respiratory microbial colonisation in our cohort. H. influenzae: Haemophilus influenzae; S. pneumoniae: Streptococcus pneumoniae; S. aureus: Staphylococcus aureus; MRSA: methicillin-resistant S. aureus; P. aeruginosa: Pseudomonas aeruginosa; M. catarrhalis: Moraxella catarrhalis; A. fumigatus: Aspergillus fumigatus; NTM: nontuberculous mycobacteria. 


\section{TABLE 1 General characteristics of adult primary ciliary dyskinesia patients}

\section{Total patients}

Age in 2014 years

Male $n(\%)$

Age at diagnosis years

Smokers n $(\%)$

Length of follow-up years

All-cause mortality $n(\%)$

Respiratory cause mortality $\mathrm{n}(\%)$

Nasal nitric oxide

Patients analysed

$\mathrm{ppb}$

$\mathrm{nL} \cdot \min ^{-1}$

\section{Ciliary beat frequency}

Patients analysed

$\mathrm{Hz}$

$\leqslant 8 \mathrm{~Hz} \%$

$>8 \mathrm{~Hz} \%$

\section{Ciliary TEM ultrastructure $\mathrm{n}(\%)$}

Patients analysed

Inner and outer dynein arm defect

Outer dynein arm defect

Inner dynein arm defect

Inner dynein arm defect with microtubular disorganisation

Central pair and transposition defect

Normal ciliary ultrastructure

Inconclusive

Ciliary TEM ultrastructure groups $\mathbf{n}(\%)$

Patients analysed

Outer arm \pm inner arm defect

Microtubular defects

Normal/inconclusive

Situs inversus $\mathbf{n}(\%)$

Patients analysed

With situs inversus

Inner and outer dynein arm defect

Outer dynein arm defect

Inner dynein arm defect

Inner dynein arm defect with microtubular disorganisation

Central pair and transposition defect

Normal ciliary ultrastructure

Unavailable

\section{HRCT scoring}

Patients analysed

Extent of bronchiectasis \%

Severity of bronchial wall dilatation \%

Bronchial wall thickness \%

Small airway mucus plugging \%

Large airway mucus plugging \%

Mosaicism \%

Emphysema \%

Pulmonary function at baseline

Patients analysed

FEV $1 \%$ pred

FEV $1 / F V C \%$

TLC \% pred

$\mathrm{RV} / \mathrm{TLC} \%$

TLCO \% pred

Kco \% pred

Cumulative sputum microbiology during follow-up period $\mathbf{n}(\%)$

Pseudomonas aeruginosa colonisation

NTM infection

Allergic bronchopulmonary aspergillosis
151

$35(19-75 ; 26-47)$

$58(38.4)$

$23.5(<1-72 ; 10-36)$

$12(8.0)$

$7(1-34 ; 3-13)$

$7(4.6)$

$5(3.3)$

97

$42.0(5.0-229.0 ; 23.0-70.0)$

$10.5(1.3-57.3 ; 5.8-17.5)$

144

$0(0-16.85)^{\#}$

80.7

19.3

141

36 (25.5)

56 (39.7)

$3(2.1)$

$14(9.9)$

$13(9.2)$

$16(11.3)$

$3(2.1)$

138

$92(66.7)$

$27(19.6)$

19 (13.8)

144

55 (38.2)

$16(29.1)$

$27(49.1)$

1 (1.8)

$4(7.3)$

$0(0)$

$3(5.5)$

4 (7.3)

93

$44.4(0-100.0 ; 33.3-63.9)$

$50.0(0.0-83.3 ; 33.3-66.7)$

$33.3(5.6-83.3 ; 22.2-44.4)$

$58.3(0.0-100.0 ; 41.7-75.0)$

$0.0(0.0-58.3 ; 0.0-0.0)$

$61.2(0.0-85.0 ; 47.5-71.7)$ $0.0(0.0-0.0)^{\#}$

118

$71.5 \pm 21.5$

$69.7 \pm 14.7$

$104.6 \pm 16.8$

$140.1 \pm 33.2$

$82.7 \pm 18.6$

$96.8 \pm 14.1$

$68(44.4)$

5 (3.3)

$4(2.6)$ 
TABLE 1 Continued

\section{Lung function change with age}

Patients analysed

Estimated change in FEV1 \% pred per year

Estimated change after adjusting for age and sex $\mathrm{mL} \cdot \mathrm{year}^{-1}$
82

$-0.49(-1.68-0.70)^{\pi}$

$-27.34(-65.13-10.44)^{\pi}$

Data are presented as median (range; interquartile range) or mean $\pm \mathrm{SD}$, unless otherwise stated. TEM: transmission electron microscopy; HRCT: high-resolution computed tomography; FEV1: forced expiratory volume in $1 \mathrm{~s}$; FVC: forced vital capacity; TLC: total lung capacity; RV: residual volume; TLCo: transfer factor of the lung for carbon monoxide; Kco: transfer coefficient of the lung for carbon monoxide, i.e. gas transfer ability per unit volume of lung; NTM: nontuberculous mycobacteria. \#: median (range); ": based on regression coefficient (estimated range where $95 \%$ of values would lie); see main text for details.

Cumulative sputum microbiological analysis revealed that $87 \%$ of patients were colonised at any point over the follow-up period. A median (interquartile range) of 12 (6-22) cultures per patient, or 2.2 (1.1-3.0) cultures per year, were collected over the follow-up period. Haemophilus influenzae (42\%) and P. aeruginosa (44\%) were the most commonly isolated organisms (figure 1c). Five (3.3\%) patients were colonised with NTM and only four (2.6\%) patients had ABPA.

There was variation in the age at diagnosis, with a median of 23.5 years (range $<1-72$ years). Baseline FEV 1 $\%$ pred correlated with age at diagnosis, with a lower FEV1 \% pred the older the patients were diagnosed $(\mathrm{r}=-0.30, \mathrm{p}=0.01)$ (figure $2 \mathrm{a}$ ). Median age at diagnosis for patients colonised with $P$. aeruginosa was significantly higher than for those not colonised with P. aeruginosa (difference in medians 17 years $(95 \%$ CI 4.5-20 years); $\mathrm{p}=0.002$ ) (figure $2 \mathrm{~b}$ ). In addition, colonisation with $P$. aeruginosa was significantly associated with decreased baseline FEV1 \% pred (difference in medians $-8.5 \%$ pred $(95 \% \mathrm{CI}-17--1 \%$ pred); $\mathrm{p}=0.02$ ) (figure 2c) and increased HRCT extent of bronchiectasis (difference in medians $-5.56 \%$ (95\% CI $-16.7-0.0 \%) ; \mathrm{p}=0.04$ ). There was no significant difference in age of diagnosis in patients with situs inversus compared with normal situs (difference in medians -11.0 years (95\% CI $-21.39--0.61$ years); $\mathrm{p}=0.27$ ) and no significant association between categories of ciliary ultrastructure and age at diagnosis, baseline $\mathrm{FEV}_{1} \%$ pred, $\mathrm{FEV}$ //forced vital capacity (FVC) ratio or HRCT scoring (supplementary table S2).

\section{Progression}

Longitudinal, formal laboratory FEV1 measures (excluding clinical spirometry for consistency) were analysed to determine disease progression. There were 603 FEV1 measures on 118 patients. Of these 118 patients, 82 patients had at least two (median 5 (interquartile range 3.0-10.25)) available FEV1 measures 1 year apart.

Lung function decline (FEV1 decline per year) in the population over the follow-up period was $-0.49 \%$ pred (95\% of patients' changes in FEV1 ranged between $-1.68 \%$ and $0.70 \%$ pred) (table 1 and figure $3 a$ ). Mixed model regression analysis revealed no significant association between longitudinal FEV1 measures and sex, BMI, ciliary beat frequency, age at diagnosis or P. aeruginosa colonisation (table 2). However, the
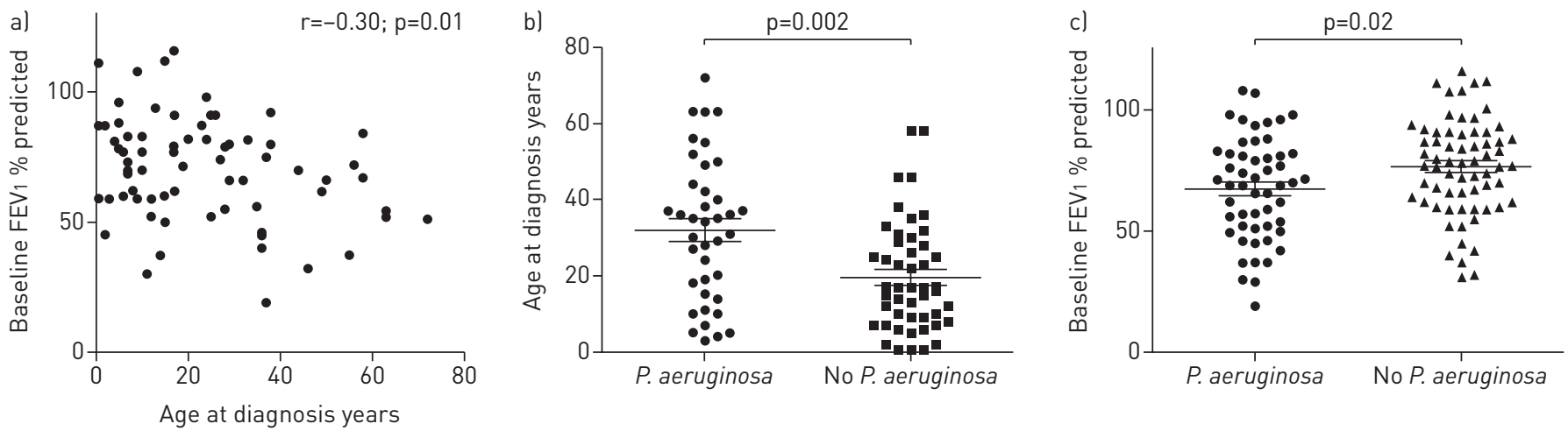

FIGURE 2 a) Correlation between age at diagnosis and baseline forced expiratory volume in $1 \mathrm{~s}$ (FEV 1 ) ( $\mathrm{r}=-0.30, p=0.01)$ in our adult primary ciliary dyskinesia cohort. b) Increased age at diagnosis was seen for those with Pseudomonas aeruginosa colonisation compared with those without (difference in medians 17 years (95\% Cl 4.5-20 years); $\mathrm{p}=0.002$ ). c) Significantly impaired FEV 1 at baseline was also seen in those with $P$. aeruginosa colonisation (difference in medians $-8.5 \%$ pred $(95 \% \mathrm{Cl}-17--1.0 \%$ pred); $\mathrm{p}=0.02)$. Horizontal lines and whiskers represent the mean and SEM, respectively. 


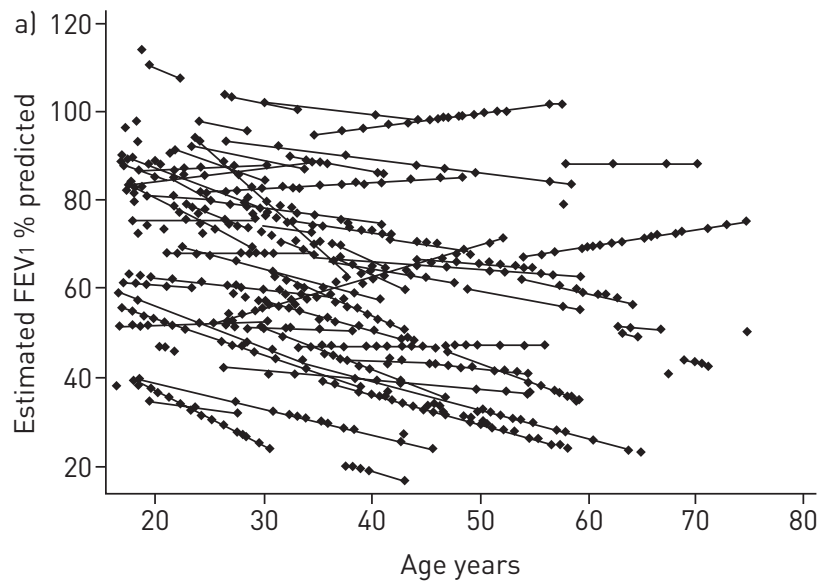

c) Extent of bronchiectasis $<44 \%$

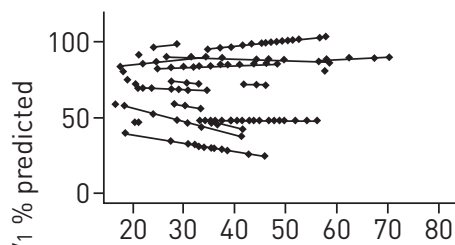

$\begin{array}{lllllll}20 & 30 & 40 & 50 & 60 & 70 & 80\end{array}$

Extent of bronchiectasis $\geqslant 55 \%$

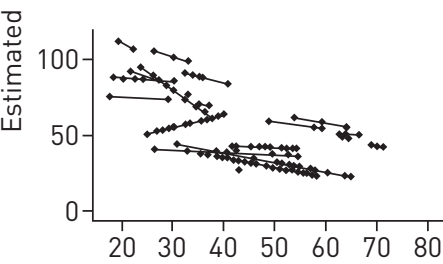

Age years

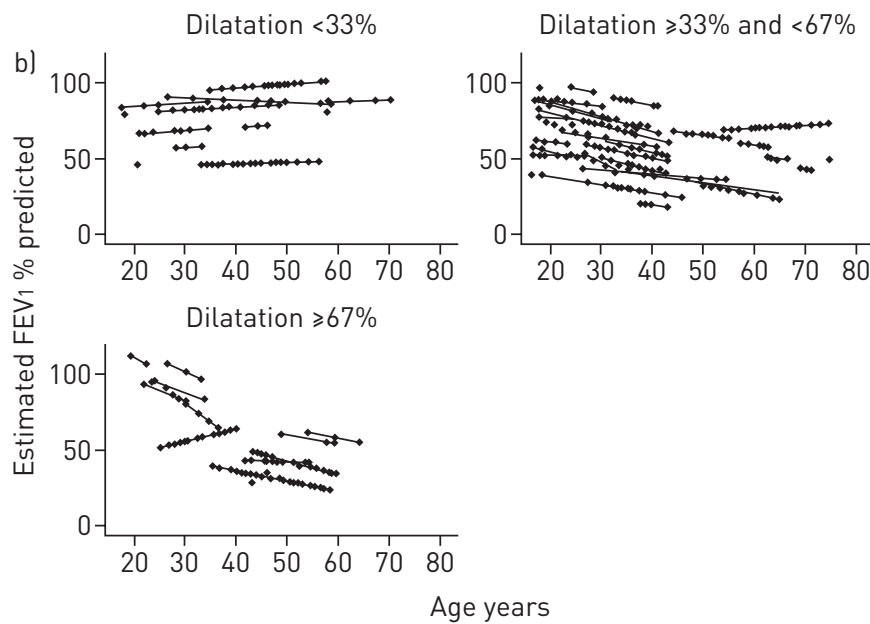

d) Microtubular defects

Normal or inconclusive
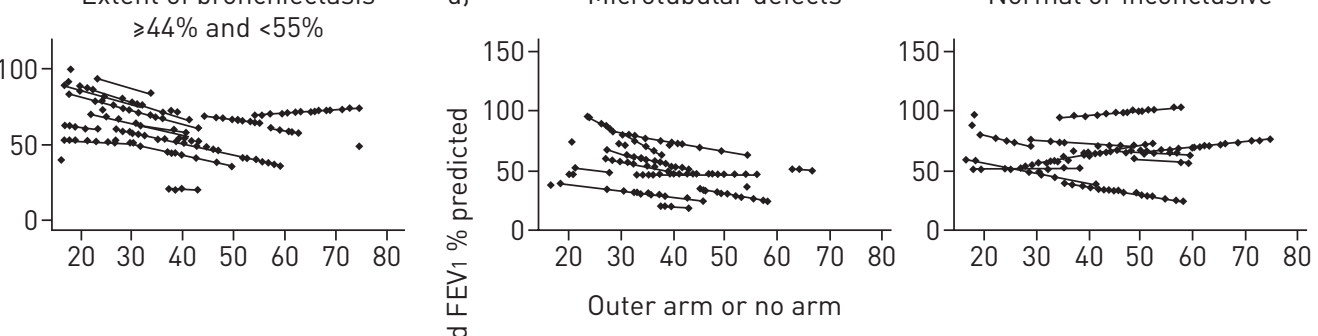

Age years

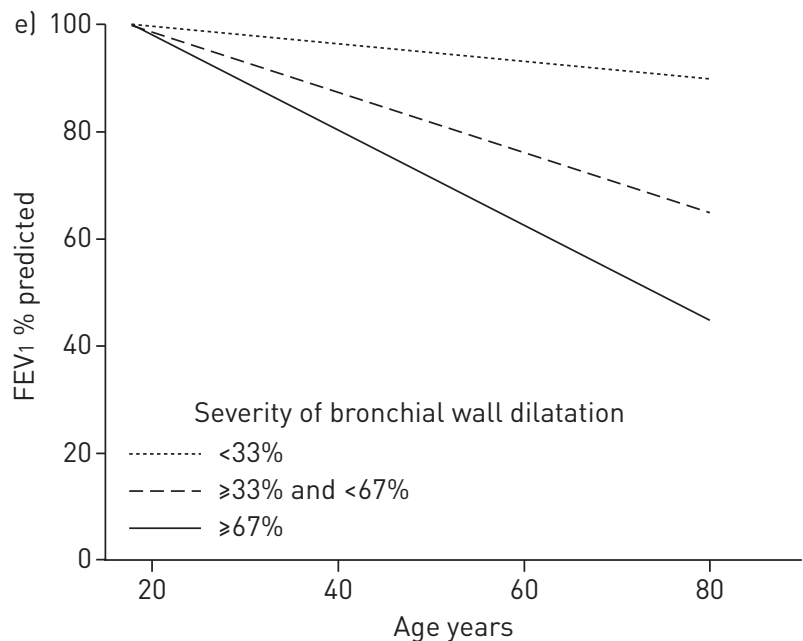

FIGURE 3 Predicted longitudinal forced expiratory volume in $1 \mathrm{~s}$ (FEV 1 ) for individual patients estimated from mixed models. Models allowed for a random effect on age. al Mixed model of the cohort adjusted for ciliary ultrastructure and age. b) Mixed model adjusting for ciliary ultrastructure and stratified by high-resolution computed tomography (HRCT) score of severity of bronchial wall dilatation. c) Mixed model adjusting for ciliary ultrastructure and stratified by HRCT score of extent of bronchiectasis. d) Mixed model stratified by ciliary ultrastructure. a-d) Predicted longitudinal FEV1 values are shown, rather than observed FEV1 values; therefore, the points follow a strict linear trend. e) Estimated projected decline calculated from mixed models within the three categories of HRCT severity of bronchial wall dilatation lusing an 18-year-old with $100 \%$ predicted FEV1 as a starting point). 
TABLE 2 Analysis of association between covariates and repeated measurements of forced expiratory volume in $1 \mathrm{~s}$ $\%$ predicted, from multilevel models

\begin{tabular}{|c|c|c|c|c|}
\hline Covariate & $\begin{array}{l}\text { B coefficient } \\
(95 \% \mathrm{CI})^{\#}\end{array}$ & p-value & $\begin{array}{l}\mathrm{p} \text {-value from likelihood ratio } \\
\text { test for interaction with age }\end{array}$ & Patients included in analysis ${ }^{+}$ \\
\hline Sex & $4.02(-4.02-12.05)$ & 0.33 & & 108 \\
\hline Age at diagnosis & $0.001(-0.35-0.35)$ & 1.00 & & 59 \\
\hline Nasal nitric oxide & $0.10(-0.01-0.22)$ & 0.09 & & 68 \\
\hline Ciliary beat frequency & $6.47(-15.76-2.82)$ & 0.17 & & 101 \\
\hline Normal or inconclusive & 0.00 & & & \\
\hline Outer \pm inner arm defect & $3.90(-7.01-14.80)$ & & & \\
\hline Microtubular defects & $10.29(-23.59-3.01)$ & & & \\
\hline Extent of bronchiectasis & $-0.18(-0.41-0.05)^{f}$ & 0.122 & 0.03 & 68 \\
\hline Severity of bronchial wall dilatation & $-0.17(-0.36-0.02)^{f}$ & 0.08 & $0.01^{+}$ & 68 \\
\hline Bronchial wall thickness & $-0.29(-0.56--0.01)^{f}$ & 0.045 & $0.06^{+}$ & 68 \\
\hline
\end{tabular}

BMI: body mass index; TEM: transmission electron microscopy. ${ }^{\#}$ : B coefficients are to be interpreted as the change in forced expiratory volume in $1 \mathrm{~s}\left(\mathrm{FEV}_{1}\right)$ for each unit change of the covariate after adjusting for age at which the measurement was made; ${ }^{\text {? }}$ tests for interaction with age assess whether these covariates affect how FEV1 changes as patients get older; ${ }^{+}$: patients included in each of these regression analyses had non-missing data for FEV1, age at the time the FEV1 measure was taken, the covariate under study, and ciliary ultrastructure where this was adjusted for; ${ }^{\S}$ : the $p$-value for this covariate relates to the covariate as a whole rather than one level or another; ${ }^{f}$ : after additionally adjusting for ciliary ultrastructure.

association was significant for ciliary ultrastructure $(\mathrm{p}=0.03)$, including when adjusted for age $(\mathrm{p}=0.04)$ (table 2). Our models were therefore stratified by categories of ciliary ultrastructure to present the estimated mean effect of age on FEV1 (table 3). We observed that patients with microtubular defects had the greatest annual FEV1 decline ( $-0.75 \%$ pred (95\% of changes ranged between $-2.08 \%$ and $0.58 \%$ pred)) compared with patients with outer or combined outer and inner arm defects $(-0.51 \%$ pred $(95 \%$ range $-1.41-0.39 \%$ pred)) and patients with normal or inconclusive TEM $(-0.13 \%$ pred (95\% range $-1.53-1.28 \%$ pred)) (figure $3 \mathrm{~d}$ and table 3 ).

TABLE 3 Mean change in forced expiratory volume in $1 \mathrm{~s}$ (FEV1) \% predicted per year in different patient subgroups, estimated from multilevel models

$\begin{array}{lcc}\text { Patient subgroups } & \begin{array}{c}\text { Mean change in } \\ \text { FEV1 \% pred per year }\end{array} & \begin{array}{c}\text { Range for } 95 \% \text { of patients' } \\ \text { changes in } \mathrm{FEV}_{1} \% \text { pred }\end{array}\end{array}$

\begin{tabular}{|c|c|c|c|}
\hline \multicolumn{4}{|c|}{ Ciliary ultrastructure by TEM ${ }^{\pi}$} \\
\hline Microtubular defects & -0.75 & $-2.08-0.58$ & 18 \\
\hline Outer \pm inner arm defect & -0.51 & $-1.41-0.39$ & 64 \\
\hline Normal or inconclusive & -0.13 & $-1.53-1.28$ & 15 \\
\hline$<44 \%$ & -0.08 & $-0.92-0.75$ & 20 \\
\hline$\geqslant 44 \%$ and $<55 \%$ & -0.65 & $-1.52-0.22$ & 25 \\
\hline$\geqslant 55 \%$ & -0.59 & $-2.13-0.95$ & 23 \\
\hline$\geqslant 33 \%$ and $<67 \%$ & -0.57 & $-1.31-0.17$ & 42 \\
\hline$\geqslant 67 \%$ & -0.89 & $-2.74-0.95$ & 14 \\
\hline \multicolumn{4}{|c|}{$\begin{array}{l}\text { TEM: transmission electron microscopy. }{ }^{\#} \text { : models were run for different patient subgroups depending on their ciliary ultrastructure, extent of } \\
\text { bronchiectasis and severity of bronchial wall dilatation; as well as this, patients were required to have non-missing data for FEV } 1 \text {, age at the } \\
\text { time the FEV } 1 \text { measure was taken and any other variables in the model. ๆ: model stratified by ciliary ultrastructure by TEM categories. } \\
+ \text { : models stratified by high-resolution computed tomography categories where there was evidence of interaction with age (p<0.05); all adjusted } \\
\text { for ciliary ultrastructure by TEM. }\end{array}$} \\
\hline
\end{tabular}


The association of HRCT characteristics with longitudinal lung function (in a model adjusting for age and ciliary ultrastructure) was analysed in 68 patients. Although severity of bronchial wall dilatation and extent of bronchiectasis were not initially associated with FEV1, they were significant when tested for interaction with age $(\mathrm{p}<0.01$ and $\mathrm{p}=0.03$, respectively) (table 2 ). Our models were therefore stratified by categories of HRCT features to present the estimated mean effect of age on FEV1. Annual change in FEV1 was $0.16 \%$ pred ( $95 \%$ of changes ranged $-0.20-0.53 \%$ pred) for $<33 \%$ bronchial wall dilatation severity, $-0.57 \%$ pred $(-1.31-0.17 \%$ pred) for $\geqslant 33 \%$ and $<67 \%$, and $-0.89 \%$ pred $(-2.74-0.95 \%$ pred $)$ for $\geqslant 67 \%$ dilatation severity. Annual change in FEV1 was $-0.08 \%$ pred $(-0.92-0.75 \%$ pred) for $<44 \%$ extent of bronchiectasis, $-0.65 \%$ pred $(-1.52-0.22 \%$ pred) for $\geqslant 44 \%$ and $<55 \%$, and $-0.59 \%$ pred $(-2.13-0.95 \%$ pred $)$ for $\geqslant 55 \%$ extent of bronchiectasis (figure $3 b, c$ and e, and table 3 ). However, the sample size was not sufficiently large to model HRCT severity score and ciliary ultrastructure subgroups independently, hence the lack of statistical significance in the slopes presented in figure 3 and table 3.

To ensure associations seen were not related to time bias, we performed a sensitivity analysis to assess the interaction between age and HRCT severity criteria only using FEV1 measures taken at or following the date of HRCT. Similar interaction with age was seen for severity of bronchial wall dilatation $(\mathrm{p}=0.04)$ within a multivariate model also adjusting for ciliary ultrastructure. We additionally explored whether HRCT scores of extent of bronchiectasis and severity of bronchial wall dilatation (both as categorical variables) modified the effect of age on other lung function parameters (see supplementary material). We observed evidence of interaction between age and severity of bronchial wall dilatation on FVC $(p=0.049)$ and on TLCO $(p=0.001)$ and interaction between extent of bronchiectasis and age on total lung capacity $(\mathrm{p}=0.01)$ and TLCO $(\mathrm{p}=0.002)$.

\section{Discussion}

In this retrospective observational study we have comprehensively characterised a large adult PCD population, associated late diagnosis with decreased FEV1 and increased $P$. aeruginosa colonisation, and identified ciliary ultrastructure and HRCT severity scores as features associated with disease progression. These findings require confirmation in prospective, systematic epidemiological studies.

In our population, we have highlighted the significant proportion of PCD patients remaining undiagnosed until adulthood despite a condition where symptoms are invariably present since early childhood [17]. There was a moderate negative correlation between age at diagnosis and FEV1 at baseline, alongside increased $P$. aeruginosa colonisation. This correlation has additionally recently been shown in preliminary data from an international multicentre PCD registry [18]. Age at diagnosis did not, however, correlate with longitudinal FEV1 measurements. This is in marked contrast to cystic fibrosis (CF), where late diagnosis is usually associated with a milder phenotype and different genetic aetiology [19]. Given the retrospective nature of this study, however, the overall impact of delayed PCD diagnosis is at present unclear and further prospective studies are required to investigate whether "optimal" management at an early stage could have an impact on subsequent disease progression.

To date, the published literature regarding outcome and longitudinal lung function in adult and paediatric PCD is conflicting, with studies showing stability in lung function over time, wide variability or steady deterioration $[2,3,20]$. In our population, overall mortality was $4.6 \%$, which reduced to $3.3 \%$ when considering respiratory-only causes of death. Prospective studies in CF with a similarly aged population have demonstrated a death or lung transplant percentage of $11 \%$ [21, 22]. Larger age-matched multicentre studies are, however, required to effectively compare cohorts. Lung function decline within our population was variable but overall relatively favourable at $-0.49 \%$ pred FEV1 per year, compared with $\mathrm{CF}$ and non-CF bronchiectasis where decline has been shown to be $-5.60 \%$ and $-2.35 \%$ pred per year, respectively $[23,24]$.

During this observational study we were able to analyse a number of variables to identify features associated with disease progression in adult PCD. The respiratory microbiota has long been thought to affect disease progression in a number of conditions, and isolation of $P$. aeruginosa has been associated with increased severity of disease and mortality in non-CF bronchiectasis and CF [22, 24-26]. Within PCD, as confirmed in our population, $P$. aeruginosa colonisation has been shown to be associated with impaired, obstructive lung function $[5,27]$. The prevalence of $P$. aeruginosa colonisation was high $(44 \%)$ compared with an older adult non-CF bronchiectasis cohort (22\%) at the Royal Brompton and Harefield NHS Foundation Trust, despite standardised disease management [25]. However, although P. aeruginosa colonisation significantly correlated with age and impaired FEV1 at diagnosis, along with radiological extent of bronchiectasis, there was no evidence of an association with longitudinal FEV1. This suggests that $P$. aeruginosa colonisation may be more a marker of disease severity in adult PCD rather than of progression; however, randomised controlled eradication trials are required for substantiation [28].

The role of ciliary ultrastructure in PCD disease progression is controversial [5]. In our population, ciliary ultrastructure profiling revealed a predominance of outer dynein arm defects, as has previously been 
shown $[4,5]$. When comparing normal ciliary ultrastructure and dynein arm defects to patients with microtubular defects, we saw that ciliary ultrastructure modified the change in FEV1 with age, with patients with microtubular defects having a greater decline (figure 3). This is in keeping with a recent multicentre paediatric cohort where patients in this group had a more severe phenotype [6]. Given the small numbers of patients within our population, however, the result should be interpreted with caution and further large multicentre cohort studies are required for confirmation. Nevertheless, the replication of findings is intriguing and further genotyping alongside ciliary and molecular phenotyping of these patients may hopefully provide further insight into the pathogenesis of different PCD subgroups.

HRCT extent of disease has been shown to be useful for predicting prognosis in a range of respiratory conditions, including non-CF bronchiectasis [29]. We explored whether HRCT severity scores would be associated with disease progression in adult PCD. Within mixed models adjusting for ciliary ultrastructure and age, examining the association between HRCT severity criteria and FEV 1 and TLCO, statistically significant interaction with age was seen with extent of bronchiectasis and severity of bronchial wall dilatation in our PCD population. This is similar to a recent small paediatric cohort study where HRCT score was found to correlate with FEV1 decline [2]. Mucus plugging, bronchial wall thickening and mosaicism were all associated with FEV1 measures but did not modify the effect of age. This is understandable with deterioration only appearing to occur once irreversible lung parenchymal changes are present on HRCT imaging. This suggests that HRCT could be used in adult PCD to identify patients at risk of disease progression for intensive management. Consequently, it is then intriguing to speculate whether aggressive management of early HRCT changes could modify subsequent disease progression.

Given the retrospective nature of this study, there are unsurprisingly significant limitations, with incomplete data, unavailable data on patient compliance and inconsistent follow-up, which could lead to risk of bias. As these data are from a tertiary referral centre, some patients may have had tests performed locally for which the results were unavailable. A further variable limitation is the use of lung function parameters alone as a marker of disease progression, as it has been suggested that HRCT imaging may be more sensitive at detecting early disease progression in CF [8, 30, 31]. Further data on quality of life and respiratory failure would provide a practical indication of the disease impact and burden in adult PCD. Due to the lack of a diagnostic gold standard in PCD, genotyping of our cohort, although not clinically available in the UK, would additionally strengthen the study. We also note that, while we did observe interaction between measures of HRCT and ciliary ultrastructure with age in the main model, the sample size was not sufficiently large to model these independently. This work was hypothesis generating and associations should be confirmed in prospective studies designed for this purpose.

Nevertheless, this population represents the largest adult PCD population characterised to date and highlights important associations and correlations requiring confirmation in larger multicentre prospective cohort studies. At present, management of adult PCD is inferred from studies in CF and non-CF bronchiectasis and, importantly, this study characterises adult PCD as a distinct disease process that requires further research and prospective therapeutic trials to identify disease-modifying treatments. We show in adult PCD that late diagnosis is associated with impairment of FEV1 at the time of diagnosis and with $P$. aeruginosa colonisation but not with longitudinal decline in lung function. We further identify ciliary ultrastructure and HRCT severity scores of extent of bronchiectasis and severity of bronchial wall dilatation as potential determinates of disease progression. This study raises further questions of the role of microtubular defects in the pathogenesis of PCD and whether identification of high-risk subgroups alongside optimal management within adult PCD following early diagnosis can achieve subsequent lung function stability.

\section{Acknowledgements}

Author contributions were as follows. A. Shah and M.R. Loebinger: conception, design, data collection and analysis, and manuscript drafting. S.J. MacNeill performed statistical analysis of the follow-up data. B. Bhaludin performed radiological scoring of HRCT images. A. Rogers and A. Shoemark performed ciliary analysis and assisted data collection. All authors contributed to the drafting of the manuscript.

\section{References}

1 Noone PG, Leigh MW, Sannuti A, et al. Primary ciliary dyskinesia: diagnostic and phenotypic features. Am J Respir Crit Care Med 2004; 169: 459-467.

2 Magnin ML, Cros $\mathrm{P}$, Beydon N, et al. Longitudinal lung function and structural changes in children with primary ciliary dyskinesia. Pediatr Pulmonol 2012; 47: 816-825.

3 Ellerman A, Bisgaard H. Longitudinal study of lung function in a cohort of primary ciliary dyskinesia. Eur Respir J 1997; 10: 2376-2379.

4 Vallet C, Escudier E, Roudot-Thoraval F, et al. Primary ciliary dyskinesia presentation in 60 children according to ciliary ultrastructure. Eur J Pediatr 2013; 172: 1053-1060.

5 Boon M, Smits A, Cuppens H, et al. Primary ciliary dyskinesia: critical evaluation of clinical symptoms and diagnosis in patients with normal and abnormal ultrastructure. Orphanet J Rare Dis 2014; 9: 11. 
Davis SD, Ferkol TW, Rosenfeld M, et al. Clinical features of childhood primary ciliary dyskinesia by genotype and ultrastructural phenotype. Am J Respir Crit Care Med 2015; 191: 316-324.

7 Maglione M, Bush A, Montella S, et al. Progression of lung disease in primary ciliary dyskinesia: is spirometry less accurate than CT? Pediatr Pulmonol 2012; 47: 498-504.

8 Irving SJ, Ives A, Davies G, et al. Lung clearance index and high-resolution computed tomography scores in primary ciliary dyskinesia. Am J Respir Crit Care Med 2013; 188: 545-549.

9 Shoemark A, Dixon M, Corrin B, et al. Twenty-year review of quantitative transmission electron microscopy for the diagnosis of primary ciliary dyskinesia. J Clin Pathol 2012; 65: 267-271.

10 Chalmers JD, Goeminne P, Aliberti S, et al. The bronchiectasis severity index. An international derivation and validation study. Am J Respir Crit Care Med 2014; 189: 576-585.

11 Griffith DE, Aksamit T, Brown-Elliott BA, et al. An official ATS/IDSA statement: diagnosis, treatment, and prevention of nontuberculous mycobacterial diseases. Am J Respir Crit Care Med 2007; 175: 367-416.

12 Pasteur MC, Bilton D, Hill AT. British Thoracic Society guideline for non-CF bronchiectasis. Thorax 2010; 65: Suppl. 1, i1-i58.

13 Quanjer PH, Stanojevic S, Cole TJ, et al. Multi-ethnic reference values for spirometry for the 3-95-yr age range: the global lung function 2012 equations. Eur Respir J 2012; 40: 1324-1343.

14 Cotes JE, Chinn DJ, Quanjer PH, et al. Standardization of the measurement of transfer factor (diffusing capacity). Report Working Party Standardization of Lung Function Tests, European Community for Steel and Coal. Official Statement of the European Respiratory Society. Eur Respir J 1993; 6: Suppl. 16, 41-52.

15 Bhalla M, Turcios N, Aponte V, et al. Cystic fibrosis: scoring system with thin-section CT. Radiology 1991; 179: $783-788$.

16 Reiff DB, Wells AU, Carr DH, et al. CT findings in bronchiectasis: limited value in distinguishing between idiopathic and specific types. AJR Am J Roentgenol 1995; 165: 261-267.

17 Coren ME, Meeks M, Morrison I, et al. Primary ciliary dyskinesia: age at diagnosis and symptom history. Acta Paediatr 2002; 91: 667-669.

18 Werner C, Lablans M, Ataian M, et al. An international registry for primary ciliary dyskinesia. Eur Respir J 2016; 47: 849-859.

19 Rodman DM, Polis JM, Heltshe SL, et al. Late diagnosis defines a unique population of long-term survivors of cystic fibrosis. Am J Respir Crit Care Med 2005; 171: 621-626.

20 Marthin JK, Petersen N, Skovgaard LT, et al. Lung function in patients with primary ciliary dyskinesia: a cross-sectional and 3-decade longitudinal study. Am J Respir Crit Care Med 2010; 181: 1262-1268.

21 de Boer K, Vandemheen KL, Tullis E, et al. Exacerbation frequency and clinical outcomes in adult patients with cystic fibrosis. Thorax 2011; 66: 680-685.

22 Goeminne PC, Nawrot TS, Ruttens D, et al. Mortality in non-cystic fibrosis bronchiectasis: a prospective cohort analysis. Respir Med 2014; 108: 287-296.

23 Que C, Cullinan P, Geddes D. Improving rate of decline of FEV1 in young adults with cystic fibrosis. Thorax 2006; 61: 155-157.

24 Martínez-García MA, Soler-Cataluña JJ, Perpiñá-Tordera M, et al. Factors associated with lung function decline in adult patients with stable non-cystic fibrosis bronchiectasis. Chest 2007; 132: 1565-1572.

25 Loebinger MR, Wells AU, Hansell DM, et al. Mortality in bronchiectasis: a long-term study assessing the factors influencing survival. Eur Respir J 2009; 34: 843-849.

26 Emerson J, Rosenfeld M, McNamara S, et al. Pseudomonas aeruginosa and other predictors of mortality and morbidity in young children with cystic fibrosis. Pediatr Pulmonol 2002; 34: 91-100.

27 Rogers GB, Carroll MP, Zain NM, et al. Complexity, temporal stability, and clinical correlates of airway bacterial community composition in primary ciliary dyskinesia. J Clin Microbiol 2013; 51: 4029-4035.

28 Davies G, Wells AU, Doffman S, et al. The effect of Pseudomonas aeruginosa on pulmonary function in patients with bronchiectasis. Eur Respir J 2006; 28: 974-979.

29 Ellis HC, Cowman S, Fernandes M, et al. Predicting mortality in bronchiectasis using bronchiectasis severity index and FACED scores: a 19-year cohort study. Eur Respir J 2016; 47: 482-489.

30 de Jong PA, Nakano Y, Lequin MH, et al. Progressive damage on high resolution computed tomography despite stable lung function in cystic fibrosis. Eur Respir J 2004; 23: 93-97.

31 Pifferi M, Caramella D, Bulleri A, et al. Pediatric bronchiectasis: correlation of HRCT, ventilation and perfusion scintigraphy, and pulmonary function testing. Pediatr Pulmonol 2004; 38: 298-303. 